Pacific Journal of Mathematics

ÜBER ABBILDUNGEN VON MENGEN 


\section{ÜBER ABBILDUNGEN VON MENGEN}

\section{Miroslav NovotnÝ}

1. Einleitung. Im Jahre 1952 habe ich folgendes Problem gelöst: Gegeben sind die Mengen $M, N$ und die Abbildung $f$ der Menge $M$ in sich und die Abbildung $g$ der Menge $N$ in sich. Es sind alle solchen Abbildungen $F$ der Menge $M$ in die Menge $N$ zu konstruieren, dass $F[f(x)]=g[F(x)]$ für jedes $x \in M$ gilt. Dieses Ergebnis erschien in einer tschechisch geschriebenen Arbeit [4], welche daher den Mathematikern schwer verständlich war. Damit lässt sich erklären, dass indessen einige Arbeiten erschienen sind, welche Spezialfälle des obigen Problems behandeln ohne meine Lösung anzuwenden: So hat sich M. W. Weaver mit dem Falle beschäftigt, in welchem $M=N, M$ endlich und $f$ eineindeutig war [5]; S. Prešić hat den Fall behandelt, in welchem $f$ eine eineindeutige Abbildung der Menge $M$ auf sich und $g$ die identische Abbildung der Menge $N$ auf sich war [3].

Das Ziel der vorliegenden Arbeit ist meine Lösung des Problems deutsch erscheinen $\mathrm{zu}$ lassen und zu zeigen, wie aus meiner Lösung die Lösung der Probleme von Weaver und von Prešic folgt. Ferner ergeben sich aus meinem Hauptsatz auch einige Resultate, die sich in Burnsides Buch [1] befinden. Ich will auch darauf hinweisen, dass aus meinem Hauptsatz die Konstruktion aller Homomorphismen einer gegebenen unären Algebra in eine gegebene unäre Algebra folgt, was ein Beitrag zu der in [2] von J. G. Marica und S. J. Bryant behandelten Problematik ist.

Übliche Begriffe und Symbolik der Mengenlehre werden als bekannt vorausgesetzt. $\varnothing$ ist die leere Menge, $\{x\}$ ist die Menge, die $x$ als einziges Element enthält. Ist $M$ eine Menge, $f$ eine Abbildung dieser Menge, $E$ eine Eigenschaft, welche die Elemente von $M$ entweder haben oder nicht haben, so ist $\{f(x) \mid E(x)\}$ die Menge aller $f(x)$, für welche $E(x)$ richtig ist. Ist $M$ eine Menge, so wird unter einer Zerlegung $Z$ auf dieser Menge ein System von nicht leeren paarweise fremden Teilmengen von $M$ verstanden, für welches $\mathrm{U}_{T \in Z} T=M$ gilt.

Ist $f$ eine Abbildung der Menge $M$ in die Menge $N, y \in N$ ein beliebiges Element, so setzen wir $f_{-1}(y)=\{x \mid x \in M, f(x)=y\}$. Ist $T \subseteq M$, $U \subseteq N$, so wird $f(T)=\{f(x) \mid x \in T\}, f_{-1}(U)=\{x \mid x \in M, f(x) \in U\}$ gesetzt. Ist $P \subseteq M$, so ist $f_{P}$ eine solche Abbildung der Menge $P$, dass $f_{P}(x)=$ $f(x)$ für jedes $x \in P$ gilt.

$\bar{P}$ ist die Mächtigkeit der Menge $P, \bar{\alpha}$ die Mächtigkeit der Ordinalzahl $\alpha$. Mit $\omega$ bezeichnen wir die kleinste unendliche Ordinalzahl.

2. Hauptsatz iiber Abbildungen. In diesem Absatz bezeichnen 
wir mit $M, N$ stets dasselbe Paar von nicht leeren Mengen, mit $\infty$ die kleinste Ordinalzahl, für welche $\bar{\infty}>\max (\overline{\bar{M}}, \overline{\bar{N}})$ ist. Es sei ferner $f$ eine Abbildung der Menge $M$ in sich, $g$ eine Abbildung der Menge $N$ in sich.

Wir bezeichnen mit $f_{0}$ die identische Abbildung der Menge $M$. Es sei $m>0$ eine beliebige ganze Zahl; wir nehmen an, dass die Abbildung $f_{m-1}$ definiert ist. Wir setzen $f_{m}(x)=f\left[f_{m-1}(x)\right]$ für jedes $x \in M$.

Definition 1. Für die Elemente $x, y \in M$ setzen wir $x \rho_{f} y$ genau dann, wenn es solche ganzen Zahlen $m \geqq 0, n \geqq 0$ gibt, dass $f_{m}(x)=$ $f_{n}(y)$ gilt. Man sieht leicht ein, dass $\rho_{f}$ eine reflexive, transitive und symetrische Relation ist; daher definiert sie eine Zerlegung $Z$ auf der Menge $M$.

\subsection{Hilfssatz. Aus $T \in Z_{f}$ folgt $f(T) \subseteq T, f_{-1}(T) \subseteq T$.}

Definition 2. Es sei $x \in M$.

(a) Wenn es zwei solche ganzen Zahlen $0 \leqq m<n$ gibt, dass $f_{m}(x)=f_{n}(x)$ ist, so ist die Menge der Elemente, welche in der Folge $f_{0}(x), f_{1}(x), f_{2}(x), \cdots, f_{k}(x), \cdots$ unendlich oft eintreten, nicht leer und endlich. Diese Menge soll Zyklus des Elementes $x$ heissen; es sei $R_{f}(x)$ die Kardinalzahl des Zyklus.

(b) Wenn die Elemente $f_{0}(x), f_{1}(x), f_{2}(x), \cdots, f_{n}(x), \cdots$ untereinander verschieden sind, so setzen wir $R_{f}(x)=0$.

Die Zahl $R_{f}(x)$ heisse Rang des Elementes $x$.

2.2. Hilfssatz. Es sei $T \in Z_{f}$. Dann haben alle Elemente $x \in T$ denselben Rang; ist überdies noch $R_{f}(x)>0$, so haben sie auch denselben Zyklus.

Beweis. Es sei $x_{1}, x_{2} \in T$. Die Folgen $f_{0}\left(x_{1}\right), f_{1}\left(x_{1}\right), f_{2}\left(x_{1}\right), \cdots$, $f_{n}\left(x_{1}\right), \cdots ; f_{0}\left(x_{2}\right), f_{1}\left(x_{2}\right), f_{2}\left(x_{2}\right), \cdots, f_{n}\left(x_{2}\right), \cdots$ haben dieselben Elemente bis auf endlich viele Ausnahmen.

Definition 3. Es sei $T \in Z_{f}$. Der gemeinsame Rang aller Elemente der Klasse $T$ wird Rang der Klasse $T$ heissen und wird mit $R_{f}(T)$ bezeichnet.

Ist $R_{f}(T)>0$, so wird der gemeinsame Zyklus aller Elemente der Klasse $T$ Zyklus der Klasse $T$ heissen.

Definition 4. Das Element $x \in M$ heisse Element mit der Eigenschaft $\pi$, wenn es eine solche Folge $\left\{x_{n}\right\}_{n<\omega}$ gibt, dass $x_{n} \in M, f\left(x_{1}\right)=x$ und $f\left(x_{n+1}\right)=x_{n}$ für jede natürliche Zahl $n$ ist. Wir bezeichnen mit $A$ die Menge aller Elemente mit der Eigenschaft $\pi$; wir setzen $B=$ 
$M-A$. Ferner setzen wir $B_{0}=\left\{x \mid x \in B, f_{-1}(x)=\varnothing\right\}$. Es sei $\alpha$ eine beliebige Ordinalzahl; wir nehmen an, dass die Menge $B_{\lambda}$ für jedes $\lambda<\alpha$ definiert ist. Wir setzen

$$
B_{\alpha}=\left\{x \mid x \in B-\mathrm{U}_{\lambda>\alpha} B_{\lambda}, f_{-1}(x) \subseteq \mathrm{U}_{\lambda<\alpha} B_{\lambda}\right\} .
$$

2.3. HILFSSATZ. Es gibt eine solche Ordinalzahl $\vartheta<\infty$, dass $B=\bigcup_{\lambda<\vartheta} B_{\lambda}$. Dabei sind die Mengen $B_{\lambda}$ zueinander fremd.

Beweis. Wir bezeichnen mit $\vartheta$ die kleinste Ordinalzahl, für welche $B_{\vartheta}=\varnothing$ ist. Es ist offenbar $\bar{\vartheta} \leqq \overline{\bar{B}}$ und $\bigcup_{\lambda<\vartheta} B_{\lambda} \leqq B$. Wäre $x \in B-$ $\bigcup_{\lambda<\vartheta} B_{\lambda}$, so gäbe es in der Menge $f_{-1}(x)$ mindestens ein Element $x_{1} \in B-$ $\mathrm{U}_{\lambda<\vartheta} B_{\lambda}$. Durch Induktion beweist man leicht, dass das Element $x$ die Eigenschaft $\pi$ hat, also ist $x \in A$, was ein Widerspruch ist.

Definition 5. Für jedes Element $x \in A$ setzen wir $S_{f}(x)=\infty$; Für jedes Element $x \in B_{\alpha}$ setzen wir $S_{f}(x)=\alpha$. Die Ordinalzahl $S_{f}(x)$ heisse Stufe des Elementes $x$.

2.4. HilfsSATZ. Aus $S_{f}(x) \neq \infty$ folgt $S_{f}[f(x)]>S_{f}(x)$; aus $S_{f}(x)=$ $\infty$ folgt $S_{f}[f(x)]=\infty$.

Beweis. Aus $S_{f}(x)=\infty$ folgt $x \in A$, also $f(x) \in A$ und $S_{f}[f(x)]=\infty$. Ist $S_{f}(x) \neq \infty, S_{f}[f(x)]=\infty$, so ist die Behauptung richtig. Es sei $S_{f}(x) \neq \infty, S_{f}[f(x)] \neq \infty$. Dann ist $S_{f}[f(x)]>S_{f}(y)$ für jedes $y \in f_{-1}[f(x)]$, also auch für $y=x$.

Definition 6. Es sei $T \in Z_{f}, x_{0} \in T$ beliebig. Wir setzen $P_{0}\left(x_{0}\right)=$ $\left\{f_{m}\left(x_{0}\right) \mid m=0,1,2, \cdots\right\}$. Wir nehmen an, dass die Mengen $P_{0}\left(x_{0}\right)$, $P_{1}\left(x_{0}\right), \cdots, P_{n}\left(x_{0}\right)$ definiert sind. Dann setzen wir

$$
P_{n+1}\left(x_{0}\right)=f_{-1}\left[P_{n}\left(x_{0}\right)\right]-\bigcup_{k=0}^{n} P_{k}\left(x_{0}\right) .
$$

2.5. HILFSSATZ. Es sei $T \in Z_{f}, x_{0} \in T$ beliebig. Es ist $T=$ $\bigcup_{n<\omega} P_{n}\left(x_{0}\right)$ und die Mengen $P_{n}\left(x_{0}\right)$ sind zueinander fremd.

Es seien $M, N$ Mengen, $f$ eine Abbildung der Menge $M$ in sich, $g$ eine Abbildung der Menge $N$ in sich. Mit $\rho_{f}$ bzw. $\rho_{g}$ wird die Relation, mit $Z_{f}$ bzw. $Z_{g}$ die Zerlegung, mit $R_{f}$ bzw. $R_{g}$ der Rang und mit $S_{f}$ bzw. $S_{g}$ die Stufe bezeichnet, welche mit Hilfe der Abbildung $f$ bzw. $g$ definiert wird.

Definition 7. Es seien $T \in Z_{f}, T^{\prime} \in Z_{g}$ Klassen. Die Klasse $T^{\prime}$ 
heisse zulässig zu der Klasse $T$ genau dann, wenn entweder

(a) $R_{g}\left(T^{\prime}\right) \neq 0, R_{g}\left(T^{\prime}\right)$ ist ein Teiler von $R_{f}(T)$, oder

(b) $R_{g}\left(T^{\prime}\right)=0, R_{f}(T)=0$ und es gibt ein Paar solcher Elemente $x_{0} \in T, x_{0}^{\prime} \in T^{\prime}$, dass $S_{f}\left[f_{m}\left(x_{0}\right)\right] \leqq S_{q}\left[g_{m}\left(x_{0}^{\prime}\right)\right]$ für jede ganze Zahl $m \geqq 0$ richtig ist.

Definition 8. Es seien $M, N$ Mengen, $f$ eine Abbildung der Menge $M$ in sich, $g$ eine Abbildung der Menge $N$ in sich, $F$ eine Abbildung der Menge $M$ in die Menge $N$. Wenn $F[f(x)]=g[F(x)]$ für jedes $x \in M$ gilt, so werden wir sagen, dass die Abbildung $F$ die Abbildung $f$ in die Abbildung g überführt.

In den Hilfssätzen 2.6, 2.7, 2.8 und 2.9 wird vorausgesetzt, dass die Abbildung $F$ die Abbildung $f$ in die Abbildung $g$ überführt.

2.6. Hilfssatz. $Z u$ jeder Klasse $T \in Z_{f}$ gibt es eine solche Klasse $T^{\prime} \in Z_{g}$, dass $F(T) \subseteq T^{\prime}$ ist.

Beweis. Ist $x^{\prime}, y^{\prime} \in F(T)$, so gibt es solche Elemente $x, y \in T$, dass $F(x)=x^{\prime}, F(y)=y^{\prime}$. Es gibt solche ganzen Zahlen $m, n \geqq 0$, dass $f_{m}(x)=f_{n}(y)$ ist. Daraus ergibt sich

$$
g_{m}\left(x^{\prime}\right)=g_{m}[F(x)]=F\left[f_{m}(x)\right]=F\left[f_{n}(y)\right]=g_{n}[F(y)]=g_{n}\left(y^{\prime}\right),
$$

woraus sich $x^{\prime} \rho_{g} y^{\prime}$ ergibt. Also gibt es eine Klasse $T^{\prime} \in Z_{g}$ mit der Eigenschaft $x^{\prime}, y^{\prime} \in T^{\prime}$. Also ist $F(T) \subseteq T^{\prime}$.

2.7. Hilfssatz. Es sei $T \in Z_{f} ; T^{\prime} \in Z_{g}$ sei die Klasse, für welche $F(T) \subseteq T^{\prime}$ ist. Dann ist entweder

(a) $R_{g}\left(T^{\prime}\right) \neq 0$ und $R_{g}\left(T^{\prime}\right)$ ist ein Teiler von $R_{f}(T)$, oder

(b) $R_{g}\left(T^{\prime}\right)=0, R_{f}(T)=0$.

Beweis. Es sei $R_{g}\left(T^{\prime}\right)>0$. Ist $R_{f}(T)=0$, so ist die Behauptung richtig. Also sei $R_{f}(T)>0$. Dann wird der Zyklus der Klasse $T$ bei der Abbildung $F$ auf den Zyklus von $T^{\prime}$ abgebildet. Daraus folgt leicht, dass $R_{g}\left(T^{\prime}\right)$ ein Teiler von $R_{f}(T)$ ist. Es sei $R_{g}\left(T^{\prime}\right)=0$. Wäre $R_{f}(T)>0$, so wäre das Bild des Zyklus von $T$ ein Zyklus in $T^{\prime}$, was ein Widerspruch ist.

\subsection{Hilfssatz. Für jedes $x \in M$ ist $S_{f}(x) \leqq S_{o}[F(x)]$.}

Beweis. Für $S_{f}(x)=0$ ist die Behauptung klar. Wir nehmen an, dass die Behauptung für jedes Element $x$ richtig ist, für welches $S_{f}(x)<\alpha \leqq \infty, \alpha>0$ gilt. Ferner nehmen wir an, dass es ein solches 
Element $x_{0}$ gibt, dass $S_{f}\left(x_{0}\right)=\alpha, S_{f}\left(x_{0}\right)>S_{g}\left[F\left(x_{0}\right)\right]$ ist. Zwei Fälle sind möglich:

(a) Es sei $\alpha<\infty$. Wir nehmen ein belieiges Element $y \in f_{-1}\left(x_{0}\right)$. Dann ist $S_{f}(y)<S_{f}\left(x_{0}\right)=\alpha$, also $S_{f}(y) \leqq S_{g}[F(y)] \leqq S_{g}\{g[F(y)]\}=$ $S_{g}\{F[f(y)]\}=S_{g}\left[F\left(x_{0}\right)\right]<S_{f}\left(x_{0}\right)=\alpha<\infty$. Daraus folgt $S_{g}[F(y)]<$ $S_{g}\{g[F(y)]\}$ nach 2.4. Aus diesen Relationen folgt $S_{f}(y) \leqq S_{g}[F(y)]<$ $S_{g}\{g[F(y)]\}=S_{g}\left[F\left(x_{0}\right)\right]$ für jedes $y \in f_{-1}\left(x_{0}\right)$. Daraus ergibt sich $S_{f}\left(x_{0}\right) \leqq$ $S_{g}\left[F\left(x_{0}\right)\right]$, was ein Widerspruch ist.

(b) Es sei $\alpha=\infty$. Dann hat $x_{0}$ die Eigenschaft $\pi$. Daraus folgt, dass $F\left(x_{0}\right)$ die Eigenschaft $\pi$ hat. Also ist $S_{g}\left[F\left(x_{0}\right)\right]=\infty=S_{f}\left(x_{0}\right)$, was ein Widerspruch ist.

Also ist die Behauptung für jedes Element $x$ richtig, für welches $S(x)=\alpha$ gilt.

2.9. Hilfssatz. Es sei $T \in Z_{f}$; es sei ferner $T^{\prime} \in Z_{g}$ die Klasse, für welche $F(T) \subseteq T^{\prime}$. Dann ist die Klasse $T^{\prime}$ zu der Klasse $T$ zulässig.

Der Beweis folgt aus 2.7 und 2.8. Man wählt $x_{0} \in T$ beliebig und setzt $x_{0}^{\prime}=F\left(x_{0}\right)$.

In den Hilfssätzen 2.10 und 2.11 und in der Definition 9 wird vorausgesetzt, dass $f$ eine Abbildung der Menge $M$ in sich und $g$ eine Abbildung der Menge $N$ in sich ist.

2.10. Hilfssatz. Es sei $T \in Z_{f}$; es sei ferner $T^{\prime} \in Z_{o}$ eine zu $T$ zulässige Klasse. Dann gibt es solche Elemente $x_{0} \in T, x_{0}^{\prime} \in T^{\prime}$, dass $S_{f}\left[f_{m}\left(x_{0}\right)\right] \leqq S_{g}\left[g_{m}\left(x_{0}^{\prime}\right)\right]$ für jede ganze Zahl $m \geqq 0$ gilt.

Beweis. Ist $R_{g}\left(T^{\prime}\right)=0$, so folgt die Behauptung aus der Definition 7. Ist $R_{g}\left(T^{\prime}\right)>0$, so genügt es das Element $x_{0}^{\prime}$ im Zyklus der Klasse $T^{\prime}$ zu wählen; es ist offenbar $S_{\circ}\left[g_{m}\left(x_{0}^{\prime}\right)\right]=\infty$ für jede ganze Zahl $m \geqq 0$.

2.11. Hilfssatz. Es sei $T \in Z_{f}$; es sei ferner $T^{\prime} \in Z_{g}$ eine $z u T$ zulässige Klasse. Es seien $y \in T, y^{\prime} \in T^{\prime}$ solche Elemente, dass $S_{f}(y) \leqq$ $S_{g}\left(y^{\prime}\right)$ gilt. Das Element $x \in f_{-1}(y)$ sei beliebig. Dann gibt es in der Menge $g_{-1}\left(y^{\prime}\right)$ mindestens ein solches Element $x^{\prime}$, dass $S_{f}(x) \leqq S_{o}\left(x^{\prime}\right)$ ist.

Beweis. Ist $S_{g}\left(y^{\prime}\right)=\infty$, so gibt es offenbar ein Element $x^{\prime} \in g_{-1}\left(y^{\prime}\right)$, mit der Eigenschaft $S_{g}\left(x^{\prime}\right)=\infty$. Es sei also $S_{f}(y) \leqq S_{g}\left(y^{\prime}\right)<\infty$. Wäre $S_{g}\left(x^{\prime}\right)<S_{f}(x)$ für jedes $x^{\prime} \in g_{-1}\left(y^{\prime}\right)$, so hätte man $S_{g}\left(x^{\prime}\right)<S_{f}(x)<$ $S_{f}[f(x)]=S_{f}(y) \leqq S_{g}\left(y^{\prime}\right)<\infty$ für jedes $x^{\prime} \in g_{-1}\left(y^{\prime}\right)$ nach 2.4. Man hätte daher $S_{g}\left(y^{\prime}\right) \leqq S_{f}(x)<S_{g}\left(y^{\prime}\right)$, was unmöglich ist. Also gibt es mindestens. ein Element $x^{\prime} \in g_{-1}\left(y^{\prime}\right)$ mit der Eigenschaft $S_{\theta}\left(x^{\prime}\right) \geqq S_{f}(x)$.

Definition 9. Konstruktion $K$. Es sei $T \in Z_{f}$ beliebig, $T^{\prime} \in Z_{\sigma}$ 
eine beliebige zu $T$ zulässige Klasse. Es seien $x_{0} \in T, x_{0}^{\prime} \in T^{\prime}$ solche Elemente, dass $S_{f}\left[f_{m}\left(x_{0}\right)\right] \leqq S_{g}\left[g_{m}\left(x_{0}^{\prime}\right)\right]$ für jede ganze Zahl $m \geqq 0$ gilt. Wir definieren $F\left[f_{m}\left(x_{0}\right)\right]=g_{m}\left(x_{0}^{\prime}\right)$ für jede ganze Zahl $m \geqq 0$; es ist dann $S_{f}(x) \leqq S_{g}[F(x)]$ für jedes Element $x \in P_{0}\left(x_{0}\right)$. Wir nehmen an, dass wir schon $F(x)$ für jedes $x \in \bigcup_{k=0}^{n} P_{k}\left(x_{0}\right)$ so definiert haben, dass $S_{f}(x) \leqq$ $S_{g}[F(x)]$ gilt. Wenn die Menge $P_{n+1}\left(x_{0}\right)$ nicht leer ist, so definieren wir die Abbildung $F$ auf dieser Menge folgendermassen:

Ist $x \in P_{n+1}\left(x_{0}\right)$, so ist $y=f(x) \in P_{n}\left(x_{0}\right)$ und das Element $F(y)=y^{\prime}$ ist definiert. Wir setzen $F(x)=x^{\prime}$, wo $x^{\prime} \in g_{-1}\left(y^{\prime}\right)$ ein beliebiges Element ist, für welches $S_{f}(x) \leqq S_{g}\left(x^{\prime}\right)$; ein solches gibt es nach 2.11.

2.12. HILFSSATZ. Die durch Konstruktion $K$ definierte Abbildung $F$ überführt die Abbildung $f_{T}$ in die Abbildung $g_{T^{\prime}}$.

Beweis. $\mathrm{Zu}$ jedem Element $x \in P_{0}\left(x_{0}\right)$ gibt es eine solche ganze Zahl $m \geqq 0$, dass $x=f_{m}\left(x_{0}\right)$ gilt. Also ist $F[f(x)]=F\left[f_{m+1}\left(x_{0}\right)\right]=g_{m+1}\left(x_{0}^{\prime}\right)=$ $g\left\{F\left[f_{m}\left(x_{0}\right)\right]\right\}=g[F(x)]$. Wir nehmen jetzt an, dass die Gleichung $F[f(x)]=g[F(x)]$ für jedes $x \in \bigcup_{k=0}^{n} P_{k}\left(x_{0}\right)$ richtig ist. Es sei $x \in P_{n+1}\left(x_{0}\right)$. Also ist $F(x)=x^{\prime} \in g_{-1}[F(y)]$, wo $y=f(x)$ ist. Daraus folgt $g[F(x)]=$ $F(y)=F[f(x)]$.

2.13. Hilfssatz. Es sei $f$ eine Abbildung der Menge $M$ in sich, $g$ eine Abbildung der Menge $N$ in sich. Es sei $T \in Z_{f}, T^{\prime} \in Z_{g}, F$ eine Abbildung, welche die Abbildung $f_{T}$ in die Abbildung $g_{T^{\prime}}$ überführt. Dann ist $F$ durch die Konstruktion $K$ definiert.

Beweis. Nach 2.9 ist die Klasse $T^{\prime}$ zu $T$ zulässig. Es sei $x_{0} \in T$ beliebig; wir setzen $x_{0}^{\prime}=F\left(x_{0}\right) \in T^{\prime}$. Dann ist $S_{f}\left[f_{m}\left(x_{0}\right)\right] \leqq S_{g}\left[g_{m}\left(x_{0}\right)\right]$ für jede ganze Zahl $m \geqq 0$ nach 2.8. Ferner ist $F\left[f_{m}\left(x_{0}\right)\right]=g_{m}\left[F\left(x_{0}\right)\right]=$ $g_{m}\left(x_{0}^{\prime}\right)$ für jede ganze Zahl $m \geqq 0$. Es sei $x \in P_{n+1}\left(x_{0}\right)$; wir setzen $y^{\prime}=$ $F[f(x)], F(x)=x^{\prime}$. Es ist $x^{\prime} \in g_{-1}\left(y^{\prime}\right)$ und $S_{f}(x) \leqq S_{g}\left(x^{\prime}\right)$ nach 2.8.

Definition 10. Es sei $M$ eine Menge, $Z$ eine Zerlegung dieser Menge. Für jedes $T \in Z$ sei $F^{T}$ eine Abbildung der Menge $T$. Wir bezeichnen mit $\bigcup_{T \in Z} F^{T}$ eine solche Abbildung $F$ der Menge $M$, dass $F(x)=F^{T}(x)$ für jedes $T \in Z$ und jedes $x \in T$ ist.

2.14. Hauptsatz. Es seien $M, N$ nicht leere Mengen, $f$ eine Abbildung der Menge $M$ in sich, $g$ eine Abbildung der Menge $N$ in sich.

(A) Es sei $\Phi$ eine solche Abbildung der Menge $Z_{f}$ in die Menge $Z_{g}$, welche zu jeder Klasse $T \in Z_{f}$ eine zu $T$ zulässige Klasse $\Phi(T) \in Z_{g}$ zuordnet. Es sei $F^{T}$ eine beliebige durch die Konstruktion $K$ definierte Abbildung der Menge $T$ in die Menge $\Phi(T)$. Dann ist $\bigcup_{\mathbb{T}_{Z_{f}}} F^{T}$ eine Abbildung der Menge $M$, welche die Abbildung $f$ in die Abbildung $g$ 
überführt.

(B) Jede Abbildung, welche die Abbildung $f$ in die Abbildung $g$ überführt, kann durch die sub (A) beschriebene Konstruktion definiert werden.

Der Beweis folgt aus 2.12, 2.6, 2.9, 2.13.

Definition 11. Es sei $M$ eine nicht leere Menge, $f, F$ Abbildungen dieser Menge in sich. Die Abbildung $F$ heisse mit der Abbildung $f$ in der Menge $M$ vertauschbar, wenn $f[F(x)]=F[f(x)]$ für jedes $x \in M$ gilt.

Wenn wir $M=N, f=g$ in 2.14 setzen, so bekommen wir

2.15. SATZ. Es sei $M$ eine nicht leere Menge, $f$ eine Abbildung dieser Menge in sich.

(A) Es sei $\Phi$ eine solche Abbildung der Menge $Z_{f}$ in sich, welche zu jeder Klasse $T \in Z_{f}$ eine zu $T$ zulässige Klasse $\Phi(T) \in Z_{f}$ zuordnet. Es sei $F^{T}$ eine beliebige durch die Konstruktion $K$ definierte Abbildung der Menge $T$ in die Menge $\Phi(T)$. Dann ist $\bigcup_{T \in Z_{f}} F$ eine mit $f$ in $M$ vertauschbare Abbildung.

(B) Jede mit $f$ in $M$ vertauschbare Abbildung kann durch die sub (A) beschriebene Konstruktion definiert werden.

3. Unäre Algebren. Es sei $A$ eine nicht leere Menge, auf der eine einstellige Operation definiert ist, d.h. eine Operation, die zu jedem Element $x \in A$ genau ein Element $x^{*} \in A$ zuordnet. Dann heisst die Menge $A$ unäre Algebra. ${ }^{1}$ Es seien $A, B$ unäre Algebren, $F$ eine Abbildung der Menge $A$ in die Menge $B$. Die Abbildung $F$ heisse Homomorphismus der Algebra $A$ in die Algebra $B$, wenn $F\left(x^{*}\right)=$ $[F(x)]^{*}$ für jedes $x \in A$ gilt.

Also ist die unäre Algebra $A$ die Menge $A$ und eine Abbildung $f$ dieser Menge in sich, welche durch die Gleichung $f(x)=x^{*}$ für jedes $x \in A$ definiert ist. Ähnlich ist die unäre Algebra $B$ die Menge $B$ und eine solche Abbildung $g$ dieser Menge in sich, dass $g(x)=x^{*}$ für jedes $x \in B$ gilt. Ein Homomorphismus $F$ der Algebra $A$ in die Algebra $B$ ist eine solche Abbildung der Menge $A$ in die Menge $B$, dass $F[f(x)]=$ $g[F(x)]$ für jedes $x \in A$ gilt; also ist $F$ eine Abbildung, welche die Abbildung $f$ in die Abbildung $g$ überführt. Offenbar ist auch jede Abbildung, welche die Abbildung $f$ in die Abbildung $g$ überführt, ein Homomorphismus der Algebra $A$ in die Algebra $B$.

Der Satz 2.14 ermöglicht zu unären Algebren $A, B$ alle Homomorhismen der Algebra $A$ in die Algebra $B$ zu konstruieren. 
4. Spezialfälle. Unter einer Permutation der Menge $M$ verstehen wir eine eineindeutige Abbildung dieser Menge auf sich. Ist $f$ eine Permutation der Menge $M$, so ist auch die Abbildung $f_{m}$ bei jeder ganzen Zahl $m \geqq 0$ eine Permutation dieser Menge; mit $f_{-m}$ werden wir die zu $f_{m}$ inverse Permutation der Menge $M$ bezeichnen. Also ist die Abbildung $f_{m}$ für jede ganze Zahl $m$ definiert.

$(\alpha)$ Es sei $M$ eine endliche Menge, $f$ eine Permutation der Menge $M$. Dann ist jede Klasse $T \in Z_{f}$ ein Zyklus und es ist daher $S_{f}(x)=\infty$ und $R_{f}(x)>0$ für jedes $x \in M$. Für jede Klasse $T \in Z_{f}$ und jedes Element $x \in T$ ist offenbar $T=P_{0}(x)$.

\section{Aus dem Satz 2.15 folgt daher:}

4.1. SATz. Es sei $M$ eine nicht leere endliche Menge, $f$ eine Permutation dieser Menge.

(A) Es sei $\Phi$ eine Abbildung der Menge $Z_{f}$ in sich, welche zu jeder Klasse $T \in Z_{f}$ eine solche Klasse $\Phi(T) \in Z_{f}$ zuordnet, dass $R_{f}[\Phi(T)]$ ein Teiler von $R_{f}(T)$ ist. Es seien $x_{r} \in T, x_{r}^{\prime} \in \Phi(T)$ bei jedem $T \in Z_{f}$ beliebige Elemente. Wir setzen $F\left[f_{m}\left(x_{r}\right)\right]=f_{m}\left(x_{T}^{\prime}\right)$ für jede ganze Zahl $m \geqq 0$. Dann ist die Abbildung $F$ auf $M$ mit $f$ vertauschbar.

(B) Jede mit $f$ vertauschbare Abbildung kann durch die sub (A) beschriebene Konstruktion definiert werden.

Das ist das Problem, welches von M. W. Weaver gelöst wurde.

Die nach (A) konstruierte Abbildung $F$ ist offenbar genau dann eine Permutation der Menge $M$, wenn die Abbildung $\Phi$ eineindeutig ist und die Klassen $T, \Phi(T)$ immer gleiche Mächtigkeiten haben. Also folgt

4.2. Satz. Es sei $M$ eine nicht leere endliche Menge, $f$ einn Permutation dieser Menge.

(A) Es sei $\Phi$ eine Permutation der Menge $Z_{f}$, welche zu jeder Klasse $T \in Z_{f}$ eine Klasse $\Phi(T) \in Z_{f}$ von gleicher Mächtigkeit zuordnet. Es seien $x_{T} \in T, x_{T}^{\prime} \in \Phi(T)$ bei jedem $T \in Z_{f}$ beliebige Elemente. Wir setzen $F\left[f_{m}\left(x_{T}\right)\right]=f_{m}\left(x_{T}^{\prime}\right)$ für jede ganze Zahl $m \geqq 0$. Dann ist $F$ eine Permutation der Menge $M$, die mit $f$ vertauschbar ist.

(B) Jede mit $f$ vertauschbare Permutation kann durch die sub (A) beschriebene Konstruktion definiert werden.

Ist $f$ in 4.2. eine reguläre Permutation der Menge $M$ (vgl. [4], S. 7), so haben alle Klassen $T \in Z_{f}$ dieselbe Mächtigkeit; also ist $\Phi$ eine beliebige Permutation der Menge $Z_{f}$. Also:

4.3. SATz. Es sei $M$ eine nicht leere endliche Menge, $f$ eine reguläre Permutation dieser Menge. 
(A) Es sei $\Phi$ eine beliebige Permutation der Menge $Z_{f}$. Es seien $x_{r} \in T, x_{T}^{\prime} \in \Phi(T)$ bei jedem $T \in Z_{f}$ beliebige Elemente. Wir setzen $F\left[f_{m}\left(x_{T}\right)\right]=f_{m}\left(x_{r}^{\prime}\right)$ für jede ganze Zahl $m \geqq 0$. Dann ist $F$ eine Permutation der Menge $M$, die mit $f$ vertauschbar ist.

(B) Jede mit $f$ vertauschbare Permutation kann durch die sub (A) beschriebene Konstruktion definiert werden.

Dieses Resultat befindet sich in Burnsides Buch [4], S. 215-216 in einer anderen Formulation.

Ist $f$ in 4.3 eine zirkuläre Permutation der Menge $M$ (vgl. [4], S. 7), so hat $Z_{f}$ nur eine Klasse $T=M$. Dann ist $x_{r} \in T, x_{r}^{\prime} \in T$ beliebig und es gibt eine solche ganze Zahl $n \geqq 0$, dass $x_{T}^{\prime}=f_{n}\left(x_{T}\right)$. Es ist $F\left[f_{m}\left(x_{r}\right)\right]=f_{m}\left(x_{r}^{\prime}\right)=f_{m}\left[f_{n}\left(x_{r}\right)\right]=f_{n}\left[f_{m}\left(x_{r}\right)\right]$ für jede ganze Zahl $m \geqq 0$; also ist $F(x)=f_{n}(x)$ für jedes Element $x \in M$. Also gilt

4.4. SATz. Es sei $M$ eine nicht leere endliche Menge, $f$ eine zirkuläre Permutation dieser Menge.

(A) $Z u$ jeder mit $f$ vertauschbaren Permutation $F$ der Menge $M$ gibt es eine solche ganze Zahl $n \geqq 0$, dass $F=f_{n}$ ist.

(B) Bei jeder ganzen Zahl $n \geqq 0$ ist $f_{n}$ mit $f$ auf $M$ vertauschbar.

Dieses Resultat befindet sich auch in Burnsides Buch [4], S. 8, Ex. 4.

$(\beta)$ Es seien $M, N$ nicht leere Mengen, $f$ eine Abbildung der Menge $M$ in sich, $i$ die identische Abbildung der Menge $N$ auf sich. Dann besteht jede Klasse der Zerlegung $Z_{i}$ aus genau einem Punkte und es ist $S_{i}(x)=\infty$ für jedes Element $x \in N$. Ist $T \in Z_{f}$ eine beliebige Klasse, so ist zu ihr jede Klasse $T^{\prime} \in Z_{i}$ zulässig. Für jedes $x \in M$ sei $T(x)$ die Klasse $T \in Z_{f}$, für welche $x \in T$ gilt. Aus 2.14 folgt:

4.5. Satz. Es seien $M, N$ nicht leere Mengen, $f$ eine Abbildung der Menge $M$ in sich.

(A) Es sei $\Phi$ eine beliebige Abbildung der Menge $Z_{f}$ in die Menge N. Wir setzen $F(x)=\Phi[T(x)]$. Dann ist

$$
F[f(x)]=F(x)
$$

für jedes $x \in M$.

(B) Jede Abbildung $F$ der Menge $M$ in die Menge $N$, welche die Bedingung (*) erfüllt, kann durch die sub (A) beschriebene Konstruktion definiert werden.

Definition 12. Es seien $M, N$ nicht leere Mengen, $f$ eine $A b-$ bildung der Menge in sich. Es sei $P$ eine solche beliebige fest gegebene Abbildung des Systems aller Teilmengen der Menge $N$ auf die Menge $N$, dass $P(\{x\})=x$ für jedes Element $x \in N$ gilt. 
(A) Es sei $\Phi$ eine beliebige Abbildung der Menge $Z_{f}$ in die Menge $N$. Für jedes $x \in M$ setzen wir $Q(x)=\Phi[T(x)]$. Dann ist $Q$ eine Abbildung der Menge $M$ in die Menge $N$. Wir setzen $Q=K(\Phi)$.

(B) Es sei $Q$ eine beliebige Abbildung der Menge $M$ in die Menge $N$. Für jedes $T \in Z_{f}$ ist $\bigcup_{x \in T}\{Q(x)\} \subseteq N$; also ist $P\left[\bigcup_{x \in T}\{Q(x)\}\right] \in N$.

Wir setzen $\Phi(T)=P\left[\bigcup_{x \in T}\{Q(x)\}\right]$. Dann ist $\Phi$ eine Abbildung der Menge $Z_{f}$ in die Menge $N$. Wir setzen $\Phi=L(Q)$.

4.6. Hilfssatz. Es seien $M, N$ nicht leere Mengen, $f$ eine Abbildung der Menge $M$ in sich, $P$ eine solche Abbildung des Systems aller Teilmengen der Menge $N$ auf die Menge $N$, dass $P(\{x\})=x$ für jedes Element $x \in N$ gilt.

Dann ist für jede Abbildung $\Phi$ der Menge $Z_{f}$ in die Menge $N$ die Formel $L[K(\Phi)]=\Phi$ richtig.

Beweis. Für jedes $T \in Z_{f}$ ist $\Phi(T)=\bigcup_{x \in T}\{\Phi[T(x)]\}=\bigcup_{x \in T}\{Q(x)\}$, wo $Q=K(\Phi)$ ist. Da die Menge $\bigcup_{x \in T}\{Q(x)\}$ genau ein Element hat, ist $\Phi(T)=P\left[\bigcup_{x \in T}\{Q(x)\}\right]=[L(Q)](T)$. Also ist $\Phi(T)=\{L[K(\Phi)]\}(T)$ für jedes $T \in Z_{f}$. Daraus ergibt sich $L[K(\Phi)]=\Phi$.

Es sei $A$ die Menge aller Abbildungen der Menge $Z_{f}$ in die Menge $N, B$ die Menge aller Abbildungen der Menge $M$ in die Menge $N$. Aus 4.6 folgt leicht, dass $L$ eine Abbildung der Menge $B$ auf die Menge $A$ ist. Um also alle Abbildungen $\Phi$ der Menge $Z_{f}$ in die Menge $N \mathrm{zu}$ bekommen genügt es in die Formel $\Phi(T)=P\left[\bigcup_{x \in T}\{Q(x)\}\right]$ alle Abbildungen $Q$ der Menge $M$ in die Menge $N$ einzusetzen. Daraus folgt leicht

4.7. SATz. Es seien $M, N$ nicht leere Mengen, $f$ eine Abbildung der Menge $M$ in sich, $P$ eine solche Abbildung des Systems aller Teilmengen der Menge $N$ auf die Menge $N$, dass $P(\{x\})=x$ für jedes $x \in N$ gilt.

(A) Es sei $Q$ eine beliebige Abbildung der Menge $M$ in die Menge N. Die durch die Gleichung

$$
F(x)=P\left[\bigcup_{t \in T(x)}\{Q(t)\}\right]
$$

definierte Abbildung $F$ genügt der Gleichung

$$
F[f(x)]=F(x) .
$$

(B) Jede Abbildung $F$ der Menge $M$ in die Menge $N$, welche die Bedingung (*) erfüllt, kann durch die sub (A) beschriebene Konstruktion definiert werden.

Wenn $f$ eine Permutation der Menge $M$ ist, so kann jede Klasse 
$T \in Z_{f}$ in der Form $\left\{f_{m}(x) \mid m=0, \pm 1, \pm 2, \cdots\right\}$ geschrieben werden, wo $x \in M$ ist. Daraus folgt

4.8. SATz. Es seien $M, N$ nicht leere Mengen, $f$ eine Permutation der Menge M, $P$ eine solche Abbildung des Systems aller Teilmengen der Menge $N$ auf die Menge $N$, dass $P(\{x\})=x$ für jedes $x \in N$ gilt.

(A) Es sei $Q$ eine beliebige Abbildung der Menge $M$ in die Menge N. Die durch die Gleichung $F(x)=P\left(\bigcup_{m=-\infty}^{+\infty}\left\{Q\left[f_{m}(x)\right]\right\}\right)^{2}$ definierte Abbildung erfüllt die Bedingung

$$
F[f(x)]=F(x)
$$

für jedes $x \in M$.

(B) Jede Abbildung $F$ der Menge $M$ in die Menge $N$, welche die Bedingung (*) erfüllt, kann durch die sub (A) beschriebene Konstruktion definiert werden.

Dieser Satz ist von S. Prešic bewiesen worden.

\section{LITERATUR}

1. W. Burnside, Theory of Groups of Finite Order, Cambridge University Press 1897.

2. J. C. Marica-S. J. Bryant, Unary algebras, Pacific J. Math., 10 (1960), 1347-1359.

3. S. Prešić, Sur l'équation fonctionelle $f(x)=f[g(x)]$. Publ. Fac. Electrotechnique Univ. Belgrade, Ser. Mat. et Phys. 64 (1961), 29-31.

4. M. Novotný, Sur un problème de la théorie des applications, Publ. Fac. Sci. Univ. Masaryk, No 344 (1953), 53-64.

5. M. W. Weaver, On the commutativity of a correspondence and a permutation, Pacific

J. Math., 10 (1960), 105-111.

${ }^{2}$ Hier haben die Symbole $+\infty,-\infty$ die übliche Bedeutung. 



\section{PACIFIC JOURNAL OF MATHEMATICS}

\section{EDITORS}

RalPh S. Phillips

Stanford University

Stanford, California

M. G. Arsove

University of Washington

Seattle 5 , Washington
J. Dugundu

University of Southern California

Los Angeles 7, California

Lowell J. Paige

University of California

Los Angeles 24, California

\section{ASSOCIATE EDITORS}
E. F. BECKENBACH
D. DERRY
H. L. ROYDEN
E. G. STRAUS
T. M. CHERRY
M. OHTSUKA
E. SPANIER
F. WOLF

\section{SUPPORTING INSTITUTIONS}

\author{
UNIVERSITY OF BRITISH COLUMBIA \\ CALIFORNIA INSTITUTE OF TECHNOLOGY \\ UNIVERSITY OF CALIFORNIA \\ MONTANA STATE UNIVERSITY \\ UNIVERSITY OF NEVADA \\ NEW MEXICO STATE UNIVERSITY \\ OREGON STATE UNIVERSITY \\ UNIVERSITY OF OREGON \\ OSAKA UNIVERSITY \\ UNIVERSITY OF SOUTHERN CALIFORNIA
}

\author{
STANFORD UNIVERSITY \\ UNIVERSITY OF TOKYO \\ UNIVERSITY OF UTAH \\ WASHINGTON STATE UNIVERSITY \\ UNIVERSITY OF WASHINGTON \\ AMERICAN MATHEMATICAL SOCIETY \\ CALIFORNIA RESEARCH CORPORATION \\ SPACE TECHNOLOGY LABORATORIES \\ NAVAL ORDNANCE TEST STATION
}

Mathematical papers intended for publication in the Pacific Journal of Mathematrcs should be typewritten (double spaced), and the author should keep a complete copy. Manuscripts may be sent to any one of the four editors. All other communications to the editors should be addressed to the managing editor, L. J. Paige at the University of California, Los Angeles 24, California.

50 reprints per author of each article are furnished free of charge; additional copses may be obtained at cost in multiples of 50 .

The Pacific Journal of Mathematics is published quarterly, in March, June, September, and December. Effective with Volume 13 the price per volume (4 numbers) is $\$ 18.00$; single issues, $\$ 5.00$. Special price for current issues to individual faculty members of supporting institutions and to individual members of the American Mathematical Society: $\$ 8.00$ per volume; single issues $\$ 2.50$. Back numbers are available.

Subscriptions, orders for back numbers, and changes of address should be sent to Pacific Journal of Mathematics, 103 Highland Boulevard, Berkeley 8, California.

Printed at Kokusai Bunken Insatsusha (International Academic Printing Co., Ltd.), No. 6 , 2-chome, Fujimi-cho, Chiyoda-ku, Tokyo, Japan.

\section{PUBLISHED BY PACIFIC JOURNAL OF MATHEMATICS, A NON-PROFIT CORPORATION}

The Supporting Institutions listed above contribute to the cost of publication of this Journal, but they are not owners or publishers and have no responsibility for its content or policies. 


\section{Pacific Journal of Mathematics}

\section{Vol. 13, No. $4 \quad$ June, 1963}

Dallas O. Banks, Bounds for eigenvalues and generalized convexity ........... 1031

Jerrold William Bebernes, A subfunction approach to a boundary value problem for

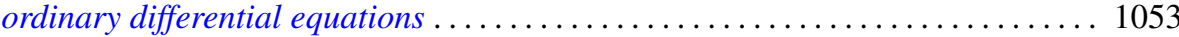

Woodrow Wilson Bledsoe and A. P. Morse, A topological measure construction . . . 1067

George Clements, Entropies of several sets of real valued functions . . . . . . . . . 1085

Sandra Barkdull Cleveland, Homomorphisms of non-commutative *-algebras . . . . . 1097

William John Andrew Culmer and William Ashton Harris, Convergent solutions of

ordinary linear homogeneous difference equations . . . . . . . . . . . . . . . 1111

Ralph DeMarr, Common fixed points for commuting contraction mappings . . . . . . 1139

James Robert Dorroh, Integral equations in normed abelian groups . . . . . . . . 1143

Adriano Mario Garsia, Entropy and singularity of infinite convolutions . . . . . . . 1159

J. J. Gergen, Francis G. Dressel and Wilbur Hallan Purcell, Jr., Convergence of extended Bernstein polynomials in the complex plane ................. 1171

Irving Leonard Glicksberg, A remark on analyticity of function algebras . . . . . . 1181

Charles John August Halberg, Jr., Semigroups of matrices defining linked operators

with different spectra ................................. 1187

Philip Hartman and Nelson Onuchic, On the asymptotic integration of ordinary

differential equations . . . . . . . . . . . . . . . . . . . . . . . . . . . . 1193

Isidore Heller, On a class of equivalent systems of linear inequalities . . . . . . . . . 1209

Joseph Hersch, The method of interior parallels applied to polygonal or multiply

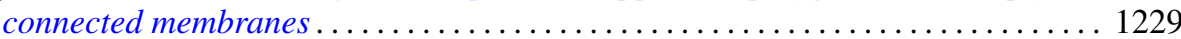

Hans F. Weinberger, An effectless cutting of a vibrating membrane . . . . . . . . . . 1239

Melvin F. Janowitz, Quantifiers and orthomodular lattices ....

Samuel Karlin and Albert Boris J. Novikoff, Generalized convex inequalities . .

Tilla Weinstein, Another conformal structure on immersed surfaces of negative

curvature.

Gregers Louis Krabbe, Spectral permanence of scalar operators

Shige Toshi Kuroda, Finite-dimensional perturbation and a representaion of

scattering operator.

Marvin David Marcus and Afton Herbert Cayford, Equality in certain

inequalities

Joseph Martin, A note on uncountably many disks .

Eugene Kay McLachlan, Extremal elements of the convex cone of semi-norms . . . . 1335

John W. Moon, An extension of Landau's theorem on tournaments . .

Louis Joel Mordell, On the integer solutions of $y(y+1)=x(x$

Kenneth Roy Mount, Some remarks on Fitting's invariants .....

Miroslav Novotný, Über Abbildungen von Mengen ............

Robert Dean Ryan, Conjugate functions in Orlicz spaces.

John Vincent Ryff, On the representation of doubly stochastic operators . . . . . . . . 1379

Donald Ray Sherbert, Banach algebras of Lipschitz functions .

James McLean Sloss, Reflection of biharmonic functions across analytic boundary

conditions with examples.

L. Bruce Treybig, Concerning homogeneity in totally ordered, connected topological space....

John Wermer, The space of real parts of a function algebra...

James Juei-Chin Yeh, Orthogonal developments of functionals and related theorems

in the Wiener space of functions of two variables......... 